\title{
Optoelectronic multiplexer for digital data processing based on liquid crystal pixels and optical fiber elements
}

\author{
I. PÉREZ, J.M.S. PENA*, J.C. TORRES, R. MANZANARES, C. MARCOS, and C. VÁZQUEZ \\ Grupo de Displays y Aplicaciones Fotónicas, Dpto. Tecnología Electrónica, Universidad Carlos III, \\ Butarque 15, E-28911 Leganés, Madrid, Spain
}

\begin{abstract}
In this work, we present an optoelectronic digital multiplexer 4:1 based on a multipixel nematic liquid crystal cell. This device uses two optical control signals to select one among four possible optical data inputs. These data signals are generated by four red LEDs, which are guided through plastic optical fiber towards liquid crystal pixels. For our purpose, only four pixels of the cell will be used to modulate the optical signal across them. Each pixel will be addressed by a square waveform coming from the conditioning circuit managed by a microcontroller system. The electronic control allows the multiplexer to work as simple two input logical gates such as AND, NAND, OR, NOR, XOR and XNOR. The operation time of the device is limited by the response time of $L C$ cell that is in the millisecond range.
\end{abstract}

Keywords: optoelectronic digital multiplexer, optical logic gate, liquid crystal, digital processing.

\section{Introduction}

Optical information systems have been the object of an extensive research activity in the last years. Historically, electronics and optical information systems were developed separately and with no significant interaction between them. Although optical systems are able to perform the process in parallel of the information, they have been limited by the difficulty of introducing or extracting the information from optical signals on real time. These factors have drastically changed in the last decades due to the raise of optoelectronics. This technological branch permits the integration between electronics and optical technology providing a new path to produce a number of devices that allow for generation, detection and optical signal control.

A relevant number of optoelectronic devices includes liquid crystals (LCs) as integrating components among many other innovative materials [1]. They have many unique and useful physical and optical properties allowing fabrication of technologically important electrooptical devices [2]. A number of interesting applications is related to the capability of handling in parallel the optical signal in optical information systems [3]. Liquid crystal devices can offer such performance although their speed of response is typically on the order of millisecond. This is not always sufficient for signal processing, but sometimes useful in such applications as low data rate information systems, or preprocessing step in hybrid computing schemes [4].

\section{Design and implementation}

The description of multiplexer components and the electronic control system are showed in the two next subsections. Unlike other proposals [5], where the control inputs of the multiplexer were electronic signals, this multiplexer can be considered an all-optical system from a functional point of view. However, several steps of optical-electronic conversion are necessary inside the system to carry out the logical information processing.

\subsection{Multiplexer design}

A twisted nematic liquid crystal display (TN-LCD) has been used to implement the 4:1 optical multiplexer (OMUX). The display is formed by a multiplexed $4 \times 4$ matrix configuration. The size of display is $10 \times 10 \mathrm{~mm}$ and the thickness is $12 \mu \mathrm{m}$. The active area of a pixel is $2.2 \times 2.2$ $\mathrm{mm}$. Each pixel of this device, placed between crossed polarizers, shows the electro-optic $(\mathrm{E} / \mathrm{O})$ response represented in Fig. 1. By applying a voltage on the pixel, liquid crystal molecules realign and rotate the polarization of the light passing through it. With a sufficient voltage, the light polarization rotates to an orthogonal state. A $1-\mathrm{kHz}$ square waveform with variable amplitude has been applied to the LC cell and $650 \mathrm{~nm}$ monochromatic light has been used in order to obtain its $\mathrm{E} / \mathrm{O}$ response. Optical transmission has been measured with a large area photodiode. Applied voltages below 1.2 Vrms maintain the pixel switched transparent while above $2.8 \mathrm{Vrms}$ keep the pixel in the dark state. Only four pixels placed in the corners of LC cell have been used as integrating electrooptical elements for the operation 


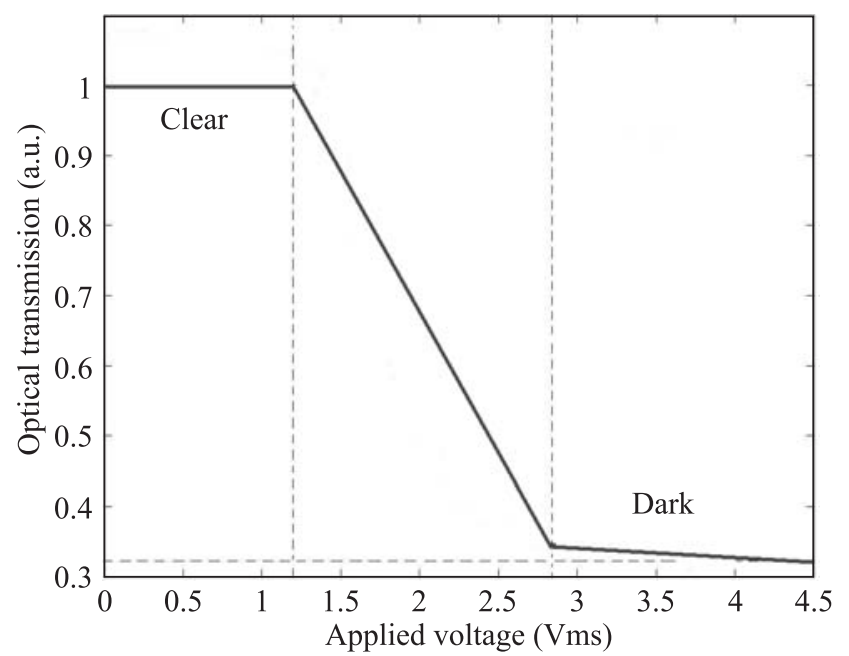

Fig. 1. Experimental electro-optic response of the TN-LCD used between crossed polarizers.

of the multiplexer. The rest of pixels always remain in the dark state.

The inputs (data and control) and the output ports of the multiplexer are made of commercial plastic optical fiber (POF). The POF (HFBR-E889328-C) has 1-mm core, a numerical aperture of 0.47 and $0.25 \mathrm{~dB} / \mathrm{m}$ losses.

Commercial plastic fiber optic red LEDs (IF-E99) supply optical data and control inputs. So, an all-optical multiplexer can be implemented by means of an optical-electronic conversion of optical control inputs. This step is necessary to switch the LC pixels that are involved in the processing of optical data supplied to the inputs of the system. Commercial plastic fiber optic phototransistors (IF-D92) and transimpedance amplifiers have been used to perform this conversion.

To obtain a 4:1 multiplexer behaviour (Fig. 2) only a pixel of the TN-LCD may be switched transparent each

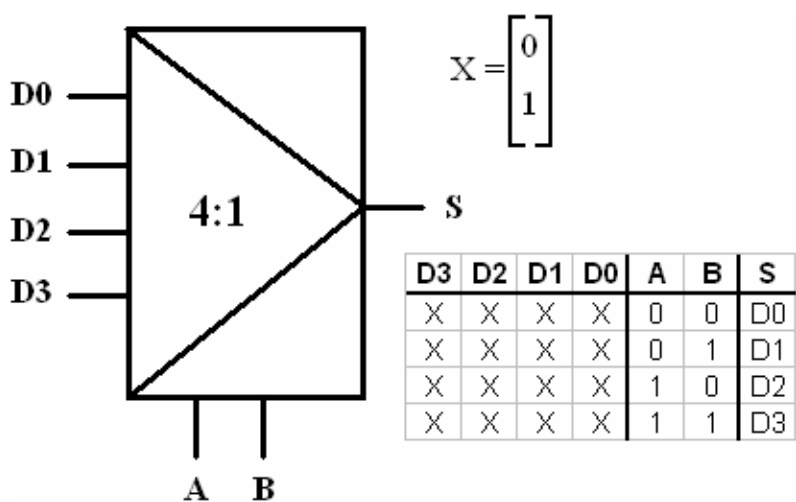

Fig. 2. Working table of a 4: 1 digital multiplexer.

time, according to the control input values. The other three pixels can remain in the dark state. To carry out this behaviour, an integrated circuit (MAX333) with four 2:1 analogue multiplexers has been used. The MAX333 supplies the appropriate voltages to the rows and columns of TN-LCD to modify the optical transmission of LC pixels.

A convergent lens has been used to couple the light coming from the LC matrix display towards the output POF of the multiplexer. A commercial plastic fiber optic phototransistor (IF-D92) and a transimpedance amplifier convert the optical output into an electric voltage: This voltage has been measured in a digital oscilloscope to check the right operation of the OMUX. Figure 3 shows the complete diagram of the developed prototype.

\subsection{Control electronics}

A digital multiplexer with appropriate width can be used to implement any combinational function. Since these logic elements can also perform sequential operations, entire digital systems can be implemented with multiplexers [5].

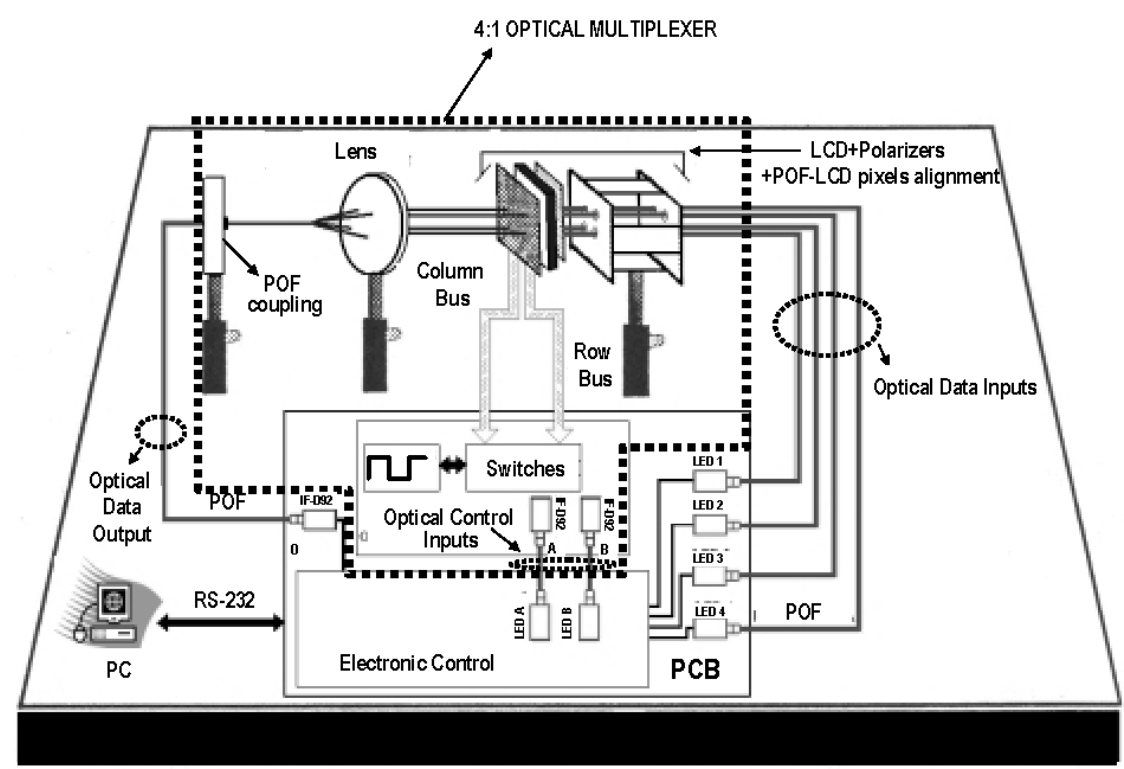

Fig. 3. Schematic diagram of developed prototype. 
In this work, an electronic control, implemented in a PCB, has been added to the optical multiplexer. This electronic control allows the multiplexer to behave like conventional two input logical gates (AND, NAND, OR, NOR, XOR, and XNOR). A software tool running on a PC has been developed for selecting the logical gate implemented and the logical value of optical signals in the inputs and output of OMUX.

The main component of the electronic control is a microcontroller device (AT89C2051). This microcontroller carries out the following tasks:

- control of multiplexer-PC communication by means of a PC series port,

- control of addressing waveforms of TN-LCD,

- control of the LEDs driving to supply four optical data inputs (light or not light) according to the logical gate selected,

- to collect the voltage at the output port of multiplexer to send the result of the selected logical function to the PC.

The software interface permits to show the selected logical function to the user, as well as the logical values for the input and output variables.
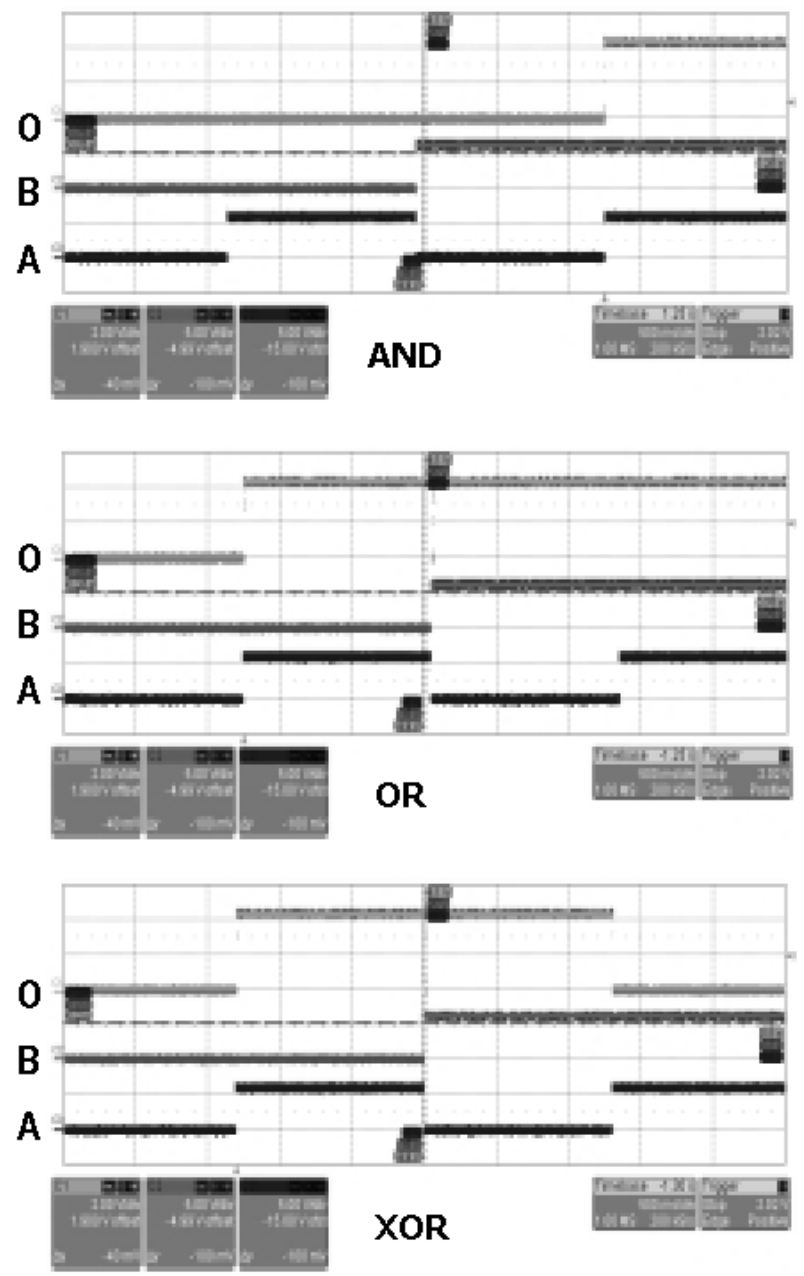

\section{Results and discussion}

A set of measurements of the two control inputs (logical function variables) of multiplexer and its output were acquired in a digital oscilloscope as it is shown in Fig. 4. Results for all logical functions checked (AND, NAND, OR, NOR, XOR and XNOR) are right. The operation time of the device is limited by the response time of LC cell that is about $5 \mathrm{~ms}$.

In this prototype, the multiplexer width could be easily increased and, therefore, more complex combinational functions could be implemented. On the other hand, combining in an appropriate way several 4:1 multiplexers, it would be very easy to implement different combinational and sequential logic systems such as conventional binary adders, comparators, line decoders, byte parity checkers/ generators, set-reset flip flops, among others [5]. This could permit its use in potential applications such as low data rate information systems, or pre-processing step in hybrid computing schemes, among others.

In order to reduce the response time of the OMUX implemented, new configurations and liquid crystals materials are being currently tested. Promising results have been ob-
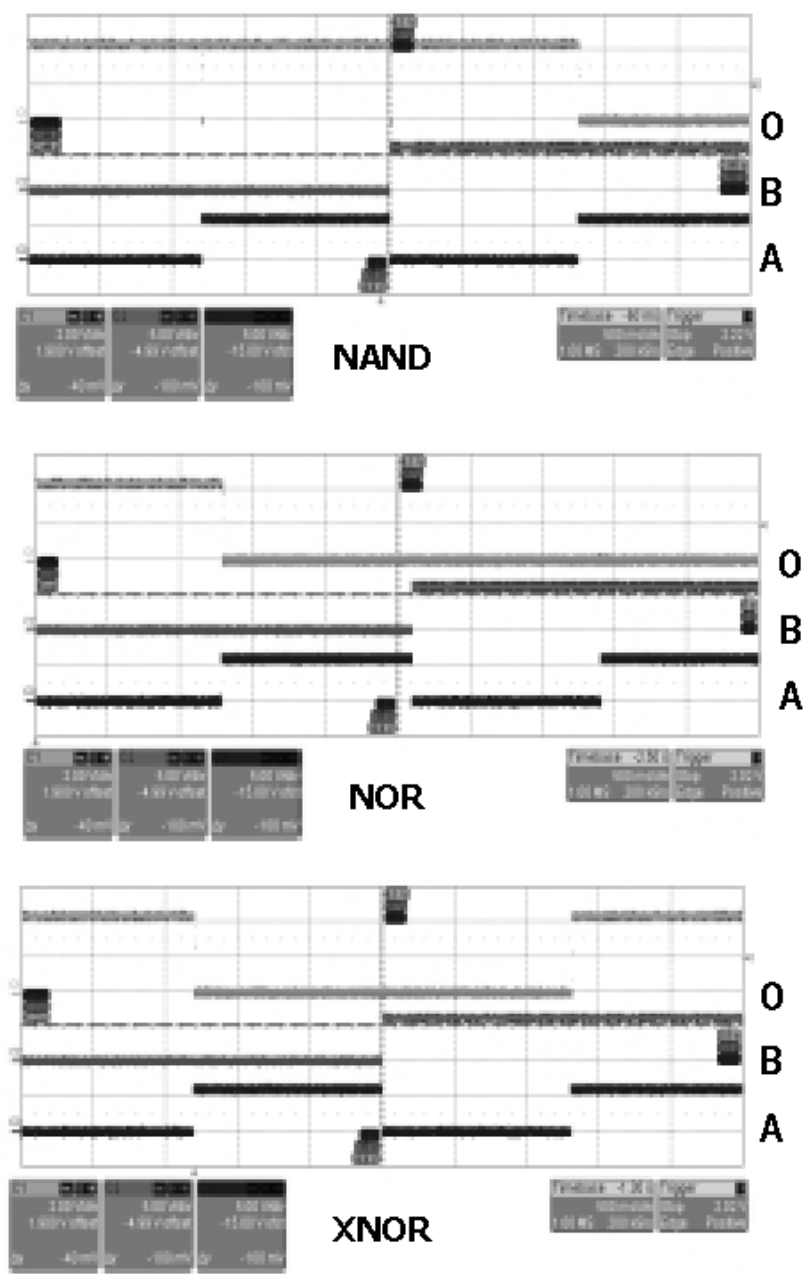

Fig. 4. Experimental results acquired in a digital oscilloscope for different logic functions. The two below traces in each plot correspond to the control inputs and the above trace is the output of the OMUX. 
tained using quiral smectic $\mathrm{C}^{*}$ liquid crystals as integrating electrooptical elements of the multiplexer. They may act as linear retarders whose direction can be switched very quickly from being along the polarization direction of incident light to being at $45^{\circ}$ from the polarization direction of the light. If these LC materials retard the light by a half wave, then the state of the light on reaching the exit polarizer may be changed from $0^{\circ}$ to $90^{\circ}$, allowing for $\mathrm{ON}$ (transparent) and OFF (opaque) operation modes. Nevertheless, the influence of the wavelength of the used optical signal could affect considerably the OMUX electrooptical performance limiting thus its use in practical applications.

\section{Conclusions}

A 4:1 optoelectronic multiplexer based on a multipixel twisted nematic LC cell is proposed. The capability of this device to implement simple logical functions operating with visible optical signals is demonstrated. The control electronics for addressing the LC pixels has been based on a microcontroller system. Operation times obtained for the most implemented logical functions are in the range of millisecond. This prototype shows a great promise in practical applications in terms of functionality and reliability. In fact, it could be integrated in more complex systems for optical processing in low data rate information systems such as optical sensor networks for automotive and dynamic reconfiguration applications in telecommunications systems, among others.

\section{Acknowledgements}

This work was partially supported by the Ministerio de Educación y Ciencia (projects TIC2003-09212-C02 and TIC2006-13392-C02-01/MIC) and Comunidad de Madrid (grant no. S-0505/ESP/000417).

\section{References}

1. J.A. Castellano, Handbook of Display Technology, Academic Press, San Diego, 1992.

2. C. Vázquez, J.M.S. Pena, I. Pérez, and S. Vargas, "Optical router for optical fiber sensor networks based on a liquid crystal cell", IEEE Sensors J. 3, 513-518 (2003).

3. R.S. McEwen., "Liquid crystals, displays and devices for optical processing", J. Phys. E: Sci. Instrum. 20, 364-377 (1987).

4. W. Klaus, Y. Suzuki, M. Tsuchiya, and T. Kamiya, "Accurate predictions of the electrooptic characteristics of homogeneous nematic crystal phase modulators", CLEO'95, Baltimore, 1995.

5. L.E.M. Brackenbury, "Multiplexer as a universal computing element for electro-optic logic systems", IEE Proc. 137, 305-310 (1990). 